\title{
Inverse Power Akash Probability Distribution with Applications
}

\author{
Samuel U. Enogwe ${ }^{1, *}$, Happiness O. Obiora-Ilouno ${ }^{2}$ and Chrisogonus K. Onyekwere ${ }^{3}$ \\ ${ }^{1}$ Department of Statistics, Micheal Okpara University of Agriculture, Umudike, Nigeria \\ e-mail: senogwe@yahoo.com \\ ${ }^{2}$ Department of Statistics, Nnamdi Azikiwe University, Awka, Nigeria \\ e-mail: obiorailounoho@yahoo.com \\ ${ }^{3}$ Department of Statistics, Nnamdi Azikiwe University, Awka, Nigeria \\ e-mail: kc.onyekwere@stu.unizik.edu.ng
}

\begin{abstract}
This paper introduces an inverse power Akash distribution as a generalization of the Akash distribution to provide better fits than the Akash distribution and some of its known extensions. The fundamental properties of the proposed distribution such as the shapes of the distribution, moments, mean, variance, coefficient of variation, skewness, kurtosis, moment generating function, quantile function, Rényi entropy, stochastic ordering and the distribution of order statistics have been derived. The proposed distribution is observed to be a heavy-tailed distribution and can also be used to model data with upside-down bathtub shape for its hazard rate function. The maximum likelihood estimators of the unknown parameters of the proposed distribution have been obtained. Two numerical examples are given to demonstrate the applicability of the proposed distribution and for the two real data sets, the proposed distribution is found to be superior in its ability to sufficiently model heavy-tailed data than Akash, inverse Akash and power Akash distributions respectively.
\end{abstract}

\section{Introduction}

In many fields of life, statistical models are formulated to analyze real-life problems and these models are commonly built using probability distributions. Several kinds of probability distributions have been used by various researchers to model different kinds of real life problems. One of such distributions is the Akash distribution developed by Received: October 28, 2020; Accepted: December 4, 2020

2010 Mathematics Subject Classification: 60E05, 62E10, 62N05.

Keywords and phrases: inverse Akash distribution, power Akash distribution, heavy-tailed data, hazard rate function, order statistics. 
[1]. If a random variable $X$ follows the Akash distribution with parameter $\theta$, then its probability density function (pdf) $g(x)$ and cumulative distribution function (cdf) $G(x)$ are respectively given by

$$
g(x)=\frac{\theta^{3}}{\theta^{2}+2}\left(1+x^{2}\right) e^{-\theta x} ; \quad x>0, \quad \theta>0
$$

and

$$
G(x)=1-\left[1+\frac{\theta x(\theta x+2)}{\theta^{2}+2}\right] e^{-\theta x} ; \quad x>0, \quad \theta>0 .
$$

The Akash distribution which is applied in modelling data with monotonic increasing hazard rate was developed as a two-component mixture of exponential $(\theta)$ and gamma $(3, \theta)$ distributions with mixing proportions $\theta^{2} /\left(\theta^{2}+2\right)$ and $2 /\left(\theta^{2}+2\right)$ respectively. The mathematical and statistical properties of the Akash distribution were equally studied by [1] and this distribution was applied in modelling datasets from the engineering and medical fields. In addition, it was shown that the Akash distribution performed better than the well-known exponential and Lindley distributions in statistical modelling. Another related study by [2] and [3] used several datasets to substantiate the claim that the Akash distribution outperforms the exponential, Lindley and Shanker distributions respectively in modelling real-life phenomena.

In recent times, several extensions and generalizations of the Akash distribution have been given in the literature. The essence of such generalizations as pointed out by [4] is to provide better fits to data and obtain more flexible models. In this regard, [5] proposed a quasi Akash distribution and defined its probability density function (pdf) as

$$
f_{1}(x)=\frac{\theta^{2}}{\alpha \theta+2}\left(\alpha+\theta x^{2}\right) e^{-\theta x} ; \quad x>0, \quad \theta>0, \quad \alpha>0 .
$$

Also, a power Akash distribution having two-parameters was developed by [6] with the following pdf

$$
f_{2}(x)=\frac{\alpha \theta^{3}}{\theta^{2}+2}\left(1+x^{2 \alpha}\right) x^{\alpha-1} e^{-\theta x^{\alpha}} ; \quad x>0, \quad \theta>0, \quad \alpha>0 .
$$

Further, [7] proposed a generalized Akash distribution with pdf 


$$
f_{3}(x)=\frac{\theta^{3}}{\theta^{2}+2 \alpha}\left(1+\alpha x^{2}\right) e^{-\theta x} ; \quad x>0, \quad \theta>0, \quad \alpha>0 .
$$

A two-parameter Akash distribution due to [8] has its pdf given by

$$
f_{4}(x)=\frac{\theta^{3}}{\theta^{2}+2 \alpha}\left(1+\alpha x^{2}\right) e^{-\theta x} ; \quad x>0, \quad \theta>0, \quad \alpha>0 .
$$

Obviously, none of the generalizations of the Akash distribution reviewed in this paper allow for modelling data with heavy tails. By heavy tails, we mean tails that decay more slowly than the tails of the normal distribution. Heavy-tailed distributions do not have all their power moments finite and quite often they do not have finite variance. As we may be aware, many datasets are heavy tailed, especially those arising from finance, actuarial science, insurance etc. The aim of this article is to introduce a heavy-tailed version of the Akash distribution, called the inverse power Akash distribution (IPAD).

\section{Derivation of the Inverse Power Akash Distribution}

Given the distribution of the Akash random variable $X$ defined in (1). Let us assume that another random $Y$ is related to $X$ by the inverse power function $Y=g(X)=X^{-\frac{1}{\alpha}}$. The derivation of the pdf of the inverse power Akash distribution entails finding the distribution of the random variable $Y$. One way to determine the pdf of $Y$ is to assume that $Y=g(X)=X^{-\frac{1}{\alpha}}$ is a one-to-one function. Next, one obtains the support of $Y=X^{-\frac{1}{\alpha}}$ as $(0, \infty)$ by solving for $y$ when $x=0$ and $x=\infty$ respectively. Suppose the observed value of $Y$ is denoted by $y=x^{-\frac{1}{\alpha}}$, then $x=y^{-\alpha}$ and $\frac{d x}{d y}=-\alpha y^{-\alpha-1}$. Plugging $x=y^{-\alpha}$ into (1) gives

$$
f\left(g^{-1}(y)\right)=\frac{\theta^{3}}{\theta^{2}+2}\left(1+y^{-2 \alpha}\right) e^{-\theta y^{-\alpha}} .
$$

As stated in [9], the probability density function of a continuous random variable $(Y)$ may be obtained using the relation

$$
f(y)=f\left(g^{-1}(y)\right)\left|\frac{d x}{d y}\right| .
$$


Consequently, the probability density function (pdf) $f(y)$ of the inverse power Akash distribution is obtained by putting (3) and $\frac{d x}{d y}=-\alpha y^{-\alpha-1}$ into (4). By so doing, we get

$$
f(y)=\frac{\alpha \theta^{3}}{\theta^{2}+2}\left(1+y^{-2 \alpha}\right) y^{-\alpha-1} e^{-\theta y^{-\alpha}} ; \quad y>0, \quad \theta>0, \quad \alpha>0 .
$$

Similarly, the cumulative density function (cdf) of the inverse power Akash distribution (IPAD) is derived as follows

$$
F(y)=P[Y \leq y]=\frac{\alpha \theta^{3}}{\theta^{2}+2} \int_{0}^{y}\left(1+x^{-2 \alpha}\right) x^{-\alpha-1} e^{-\theta x^{-\alpha}} d x .
$$

Substituting $y=x^{-\frac{1}{\alpha}}, x=y^{-\alpha}$ and $\frac{d x}{d y}=-\alpha y^{-\alpha-1}$ into (6) yields

$$
F(y)=\frac{\theta^{3}}{\theta^{2}+2} \int_{y^{-\alpha}}^{\infty}\left(1+y^{2}\right) e^{-\theta y} d y
$$

Integrating Eq. (7) by parts leads to

$$
F(y)=\frac{\theta^{3}}{\theta^{2}+2}\left[\frac{1}{\theta}+\frac{y^{-2 \alpha}}{\theta}+\frac{2 y^{-2 \alpha}}{\theta^{2}}+\frac{2}{\theta^{3}}\right] e^{-\theta y^{-\alpha}} .
$$

Further simplification of (8) yields the cdf $F(y)$ of the inverse power Akash distribution as

$$
F(y)=\left[1+\frac{\theta y^{-\alpha}\left(\theta y^{-\alpha}+2\right)}{\theta^{2}+2}\right] e^{-\theta y^{-\alpha}} ; \quad y>0, \quad \alpha>0, \quad \theta>0 .
$$

Notably, the IPAD defined in (5) is also a two-component mixture of the inverse Weibull distribution (with shape parameter $\alpha$ and scale parameter $\theta$ ) and a generalized inverse gamma distribution (with shape parameters 3 and $\alpha$, and scale parameter $\theta$ ) with mixing proportions $\theta^{2} /\left(\theta^{2}+2\right)$ and $2 /\left(\theta^{2}+2\right)$. The IPAD may be called a generalized Inverse Akash (GIA) distribution because for $\alpha=1$, the distribution reduces to the 
inverse Akash distribution with pdf

$$
f(y)=\frac{\theta^{3}}{\theta^{2}+2}\left(1+y^{-2}\right) y^{-2} e^{-\theta y^{-1}} ; \quad y>0, \quad \theta>0 .
$$

Interestingly, the inverse Akash distribution offers more flexibility than the Akash distribution since it can be used to model datasets with heavy-tailed data as well as bathtub and upside-down bathtub shapes for its failure rate. It may be noted that the inverse power Akash distribution proposed in this work has polynomial tails for all values of $\alpha$ and $\theta$, and so can be referred to as a heavy-tailed distribution. In this distribution, $\alpha$ is called the heavy tailed parameter, which controls the rate of decay of the upper tail. The larger the value of $\alpha$, the less heavy the rate of decay of the upper tail. The smaller the value of $\alpha$, the more heavy the tail becomes.

\section{Investigation of the Proposed Inverse Power Akash Distribution for Proper Density Function}

According to [9], a function $f(y)$ is said to be a proper density function of a random variable $Y$ if and only if $f(y) \geq 0, \forall y$ and the total area under the curve of $f(y)$ is unity. Cases 1 and 2 below verify these conditions for validity of a pdf:

Case 1. $f(y)=\frac{\alpha \theta^{3}}{\theta^{2}+2}\left(1+y^{-2 \alpha}\right) y^{-\alpha-1} e^{-\theta y^{-\alpha}}>0$ for all $y$.

Case 2. The property that total area under the curve of $f(y)$ is unity implies that $\int_{-\infty}^{\infty} f(y) d y=1$. Thus,

$$
\begin{aligned}
\int_{0}^{\infty} f(y) d y & =\frac{\alpha \theta^{3}}{\theta^{2}+2} \int_{0}^{\infty}\left(1+y^{-2 \alpha}\right) y^{-\alpha-1} e^{-\theta y^{-\alpha}} d y \\
& =\frac{\theta^{3}}{\theta^{2}+2} \int_{0}^{\infty}\left(1+x^{2}\right) e^{-\theta x} d x \quad \because y=x^{-\frac{1}{\alpha}}, x=y^{-\alpha} \text { and } \frac{d x}{d y}=-\alpha y^{-\alpha-1} \\
& =\frac{\theta^{3}}{\theta^{2}+2}\left[\frac{\Gamma(1)}{\theta}+\frac{\Gamma(3)}{\theta^{3}}\right]=\frac{\theta^{3}}{\theta^{2}+2}\left[\frac{1}{\theta}+\frac{2}{\theta^{3}}\right]=1 .
\end{aligned}
$$


The two cases above justify that the inverse power Akash distribution proposed in this paper is indeed a valid probability density function (pdf).

The rest of the paper has been organized in the following sections. Sections 4 and 5 provide discussion of some properties of the IPAD and the reliability analysis. Entropy measure and stochastic ordering are derived in Sections 6 and 7 respectively. The maximum likelihood estimators of the unknown parameters of the proposed distribution are given in Section 8. The asymptotic distribution and the approximate confidence intervals of the maximum likelihood estimators of the IPAD are derived in Section 9. Further, the distributions of order statistics are derived in Section 10. Section 11 presents the application of the distribution to the fit attained by some other competing distributions. The paper is concluded in Section 12.

\section{Properties of the Inverse Power Akash Distribution}

In this section, some mathematical and statistical properties of the IPAD are examined. These properties are of vital importance especially when applying the distribution in analyzing real life data.

\subsection{Shape of the inverse power Akash distribution}

Once data have been collected for statistical modelling, it is necessary to determine the shape of the statistical distribution proposed for the modelling. The shape of the distribution enables one to know whether or not the proposed method is appropriate for analyzing the data. To describe the shape of the inverse power Akash probability distribution defined in (1), there is need to obtain the first derivative of the pdf. Thus,

$$
f^{1}(y)=\frac{d}{d y} f(y)=\frac{\alpha \theta^{3}}{\theta^{2}+2} y^{-\alpha-2} e^{-\theta y^{-\alpha}} \phi\left(y^{-\alpha}\right),
$$

where $\phi(\omega)=\alpha \theta \omega^{3}-(2 \alpha+\alpha+1) \omega^{2}+\alpha \theta \omega-(\alpha+1)$ and $y^{-\alpha}=\omega$. Notably, the mode of the proposed distribution is the value of $y^{-\alpha}$ for which $f^{1}(y)=0$. From (10), $f^{1}(y)=0$ implies that only $\phi(\omega)=0$. One is then required to find the zeroes of $\phi(\omega)=0$. To this end, we write $\phi(\omega)=0$ as

$$
\omega^{3}+a_{2} \omega^{2}+a_{1} \omega+a_{0}=0 .
$$

Defining $u=\omega+a_{2} / 3$ and $\omega=u-a_{2} / 3$ in (11), one obtains 


$$
u^{3}+a u+b=0
$$

where

$$
\begin{aligned}
a & =a_{1}-a_{2}^{2} / 3=1-(3+1 / \alpha)\left(3 \theta^{2}\right)^{-1} \\
b & =a_{0}-\left(a_{2} a_{1} / 3+2 a_{2}^{2} / 27\right) \\
& =-(1+1 / \alpha) \theta^{-1}+(3+1 / \alpha)\left[(3 \theta)^{-1}+2(3+1 / \alpha)\left(27 \theta^{2}\right)^{-1}\right] .
\end{aligned}
$$

The solution of (12) is obtained using the formula

$$
u=A+B
$$

where

$$
A=\left(-\frac{b}{2}+\sqrt{\frac{b^{2}}{4}+\frac{a^{3}}{27}}\right)^{\frac{1}{3}} \text { and } B=\left(-\frac{b}{2}-\sqrt{\frac{b^{2}}{4}+\frac{a^{3}}{27}}\right)^{\frac{1}{3}}
$$

After the value of $u$ has been obtained, it has to be substituted into the relation $\omega=u-a_{2} / 3$ to get the value of $\omega$. Finally, the value of $y$ is obtained from the relation $y^{-\alpha}=\omega$, and this value of $y$ is the mode of the inverse power Akash random variable.

\subsection{Asymptotic behaviour of the proposed distribution}

The asymptotic behaviour of a distribution is investigated by evaluating the limit of the probability density function (pdf) as the observed values of the random variable tends to zero and infinity respectively. As orchestrated by [10], if $\lim _{y \rightarrow 0} f(y)=0$ and $\lim _{y \rightarrow \infty} f(y)=0$, then a distribution has at least one mode. Hence, for the inverse power Akash distribution, it is obvious that $f(0)=0$ and $f(+\infty)=0$, which implies that the proposed distribution is always unimodal. Figures 1 and 2 shows various shapes of the probability density function (pdf) and that of the cumulative distribution function (cdf) of the IPAD for different values of $\theta$ and $\alpha$. 


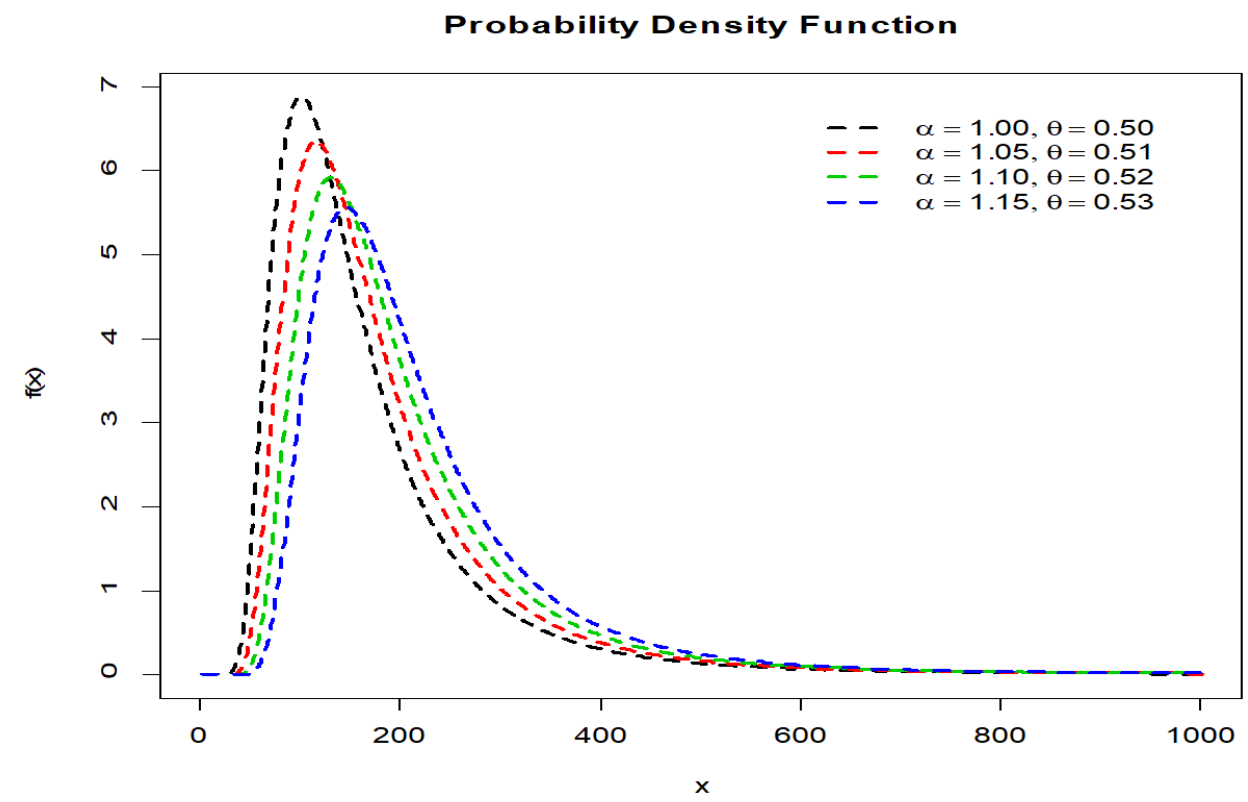

Figure 1. Shape of the pdf of the IPAD for various values of parameters $\theta$ and $\alpha$.

\section{Cummulative Density Function}

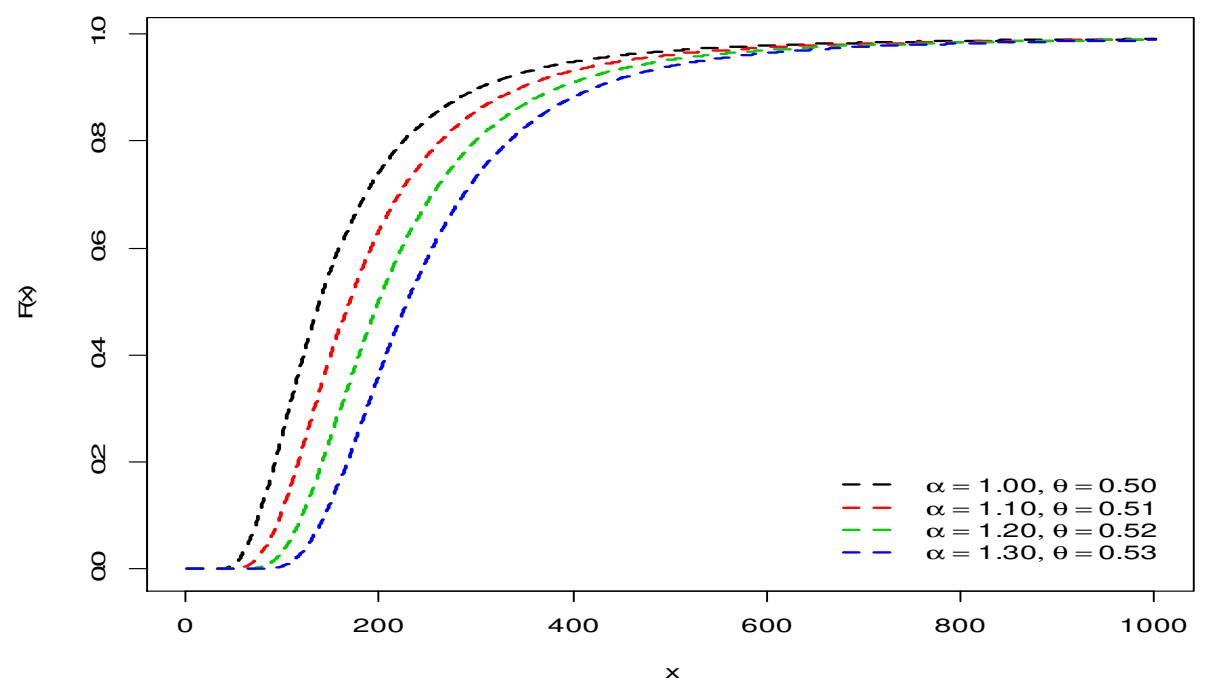

Figure 2. Shape of the cdf of the IPAD for various values of parameters $\theta$ and $\alpha$. 
The asymptotics of the pdf, cdf and the upper tails of the pdf are polynomials (i.e., heavy upper tails), while its lower tails decay exponentially. The mode moves more to the right and the pdf becomes more peaked with increasing values of $\theta$.

\subsection{Quantile function of the Inverse power Akash distribution}

The $p$ th quantile of the IPAD is the value of $y$ that satisfies the equation

$$
\begin{aligned}
p & =F(y) \\
\Rightarrow & p=\left(1+\frac{\theta y^{-\alpha}\left(\theta y^{-\alpha}+2\right)}{\theta^{2}+2}\right) e^{-\theta y^{-\alpha}} .
\end{aligned}
$$

On solving (14) for $y$, we get

$$
y_{p}=\left[\frac{1}{\theta} \ln \left(\frac{\theta^{2}+\theta y_{p}^{-\alpha}\left(\theta y_{p}^{-\alpha}+2\right)+2}{p\left(\theta^{2}+2\right)}\right)\right]^{-\frac{1}{\alpha}} .
$$

Undoubtedly, the quantile function can be useful for random number generation, estimation based on percentiles and quantile regression methods. For random number generation, one can solve (15) for $y_{p}$ for $p$ a uniform random number between 0 and 1 . The solving must be performed numerically, for example, using uniroot in the R software [11].

\subsection{Moments of the inverse power Akash distribution}

The moments of distributions are used to describe some of the most important features of a model like dispersion, skewness and kurtosis. Consequently, the $r$ th raw moment of the IPAD is given by

$$
\begin{aligned}
\mu_{r}^{1} & =E\left(Y^{r}\right)=\int_{0}^{\infty} y^{r} \frac{\alpha \theta^{3}}{\theta^{2}+2}\left(1+y^{-2 \alpha}\right) y^{-\alpha-1} e^{-\theta y^{-\alpha}} d y \\
& =\frac{\alpha \theta^{3}}{\theta^{2}+2}\left[\int_{0}^{\infty} \frac{e^{-\theta / y^{\alpha}}}{y^{\alpha-r+1}} d y+\int_{0}^{\infty} \frac{e^{-\theta / y^{\alpha}}}{y^{3 \alpha-r+1}} d y\right] .
\end{aligned}
$$


Putting $y=x^{-\frac{1}{\alpha}}, x=y^{-\alpha}$ and $\frac{d x}{d y}=-\alpha y^{-\alpha-1}$ into (16), one gets

$$
\begin{aligned}
& \mu_{r}^{1}=E\left(Y^{r}\right)=\frac{\theta^{3}}{\theta^{2}+2}\left[\int_{0}^{\infty} \frac{e^{-\theta / x}}{x^{\left(1-\frac{r}{\alpha}\right)+1}} d x+\int_{0}^{\infty} \frac{e^{-\theta / x}}{x^{\left(3-\frac{r}{\alpha}\right)+1}} d x\right] \\
& =\frac{\theta^{3}}{\theta^{2}+2}\left[\frac{\Gamma\left(1-\frac{r}{\alpha}\right)}{\theta^{\left(1-\frac{r}{\alpha}\right)}}+\frac{\Gamma\left(3-\frac{r}{\alpha}\right)}{\theta^{\left(3-\frac{r}{\alpha}\right)}}\right] \because \int_{0}^{\infty} \frac{e^{-\beta / w}}{w^{\alpha+1}} d w=\frac{\Gamma(\alpha)}{\beta^{\alpha}} \\
& =\frac{\theta^{3} \theta^{\frac{r}{\alpha}}}{\theta^{2}+2}\left[\frac{\Gamma\left(1-\frac{r}{\alpha}\right)}{\theta}+\frac{\left(1-\frac{r}{\alpha}\right)\left(2-\frac{r}{\alpha}\right) \Gamma\left(1-\frac{r}{\alpha}\right)}{\theta^{3}}\right] \because \Gamma(\alpha)=(\alpha-1) \Gamma(\alpha-1) \\
& \therefore \mu_{r}^{1}=\theta^{\frac{r}{\alpha}}\left[1+\frac{r(r-3 \alpha)}{\alpha^{2}\left(\theta^{2}+2\right)}\right] \Gamma\left(\frac{\alpha-r}{\alpha}\right), \quad \alpha>r \text {. }
\end{aligned}
$$

On substituting $r=1,2,3$ and 4 into (17), one gets the first four crude moments of the IPAD as

$$
\begin{aligned}
& \mu_{1}^{1}=\theta^{\frac{1}{\alpha}}\left[1+\frac{(1-3 \alpha)}{\alpha^{2}\left(\theta^{2}+2\right)}\right] \Gamma\left(\frac{\alpha-1}{\alpha}\right), \quad \alpha>1 \\
& \mu_{2}^{1}=\theta^{\frac{2}{\alpha}}\left[1+\frac{2(2-3 \alpha)}{\alpha^{2}\left(\theta^{2}+2\right)}\right] \Gamma\left(\frac{\alpha-2}{\alpha}\right), \quad \alpha>2 \\
& \mu_{3}^{1}=\theta^{\frac{3}{\alpha}}\left[1+\frac{3(3-3 \alpha)}{\alpha^{2}\left(\theta^{2}+2\right)}\right] \Gamma\left(\frac{\alpha-3}{\alpha}\right), \quad \alpha>3 \\
& \mu_{4}^{1}=\theta^{\frac{4}{\alpha}}\left[1+\frac{4(4-3 \alpha)}{\alpha^{2}\left(\theta^{2}+2\right)}\right] \Gamma\left(\frac{\alpha-4}{\alpha}\right), \quad \alpha>4 .
\end{aligned}
$$

The $r$ th central moment of the IPAD is given by

$$
\mu_{r}=E(X-\mu)^{r}=\sum_{j=1}^{r}\left(\begin{array}{l}
r \\
j
\end{array}\right)(-\mu)^{r-j} E\left(X^{j}\right)
$$




$$
=\sum_{j=1}^{r}\left(\begin{array}{l}
r \\
j
\end{array}\right)\left\{-\theta^{\frac{1}{\alpha}}\left[1+\frac{(1-3 \alpha)}{\alpha^{2}\left(\theta^{2}+2\right)}\right] \Gamma\left(\frac{\alpha-1}{\alpha}\right)\right\}^{r-j} \theta^{\frac{j}{\alpha}}\left[1+\frac{j(j-3 \alpha)}{\alpha^{2}\left(\theta^{2}+2\right)}\right] \Gamma\left(\frac{\alpha-j}{\alpha}\right) .
$$

In particular, the second, third and fourth central moments of the inverse power Akash distribution are

$$
\begin{aligned}
& \mu_{2}=\sum_{j=1}^{2}\left(\begin{array}{l}
2 \\
j
\end{array}\right)\left\{-\theta^{\frac{1}{\alpha}}\left[1+\frac{(1-3 \alpha)}{\alpha^{2}\left(\theta^{2}+2\right)}\right] \Gamma\left(\frac{\alpha-1}{\alpha}\right)\right\}^{2-j} \theta^{\frac{j}{\alpha}}\left[1+\frac{j(j-3 \alpha)}{\alpha^{2}\left(\theta^{2}+2\right)}\right] \Gamma\left(\frac{\alpha-j}{\alpha}\right) \\
& \mu_{3}=\sum_{j=1}^{3}\left(\begin{array}{l}
3 \\
j
\end{array}\right)\left\{-\theta^{\frac{1}{\alpha}}\left[1+\frac{(1-3 \alpha)}{\alpha^{2}\left(\theta^{2}+2\right)}\right] \Gamma\left(\frac{\alpha-1}{\alpha}\right)\right\}^{3-j} \theta^{\frac{j}{\alpha}}\left[1+\frac{j(j-3 \alpha)}{\alpha^{2}\left(\theta^{2}+2\right)}\right] \Gamma\left(\frac{\alpha-j}{\alpha}\right) \\
& \mu_{4}=\sum_{j=1}^{4}\left(\begin{array}{l}
4 \\
j
\end{array}\right)\left\{-\theta^{\frac{1}{\alpha}}\left[1+\frac{(1-3 \alpha)}{\alpha^{2}\left(\theta^{2}+2\right)}\right] \Gamma\left(\frac{\alpha-1}{\alpha}\right)\right\}^{4-j} \theta^{\frac{j}{\alpha}}\left[1+\frac{j(j-3 \alpha)}{\alpha^{2}\left(\theta^{2}+2\right)}\right] \Gamma\left(\frac{\alpha-j}{\alpha}\right) .
\end{aligned}
$$

Notably, the first raw moment $\left(\mu_{1}^{1}\right)$ is the mean $(\mu)$ while the second central moment $\left(\mu_{2}\right)$ is the variance $\left(\sigma^{2}\right)$ of the inverse power Akash distribution. It is often more convenient to write the variance as $\sigma^{2}=E\left(Y^{2}\right)-[E(Y)]^{2}$. Consequently,

$$
\sigma^{2}=\theta^{\frac{2}{\alpha}}\left[1+\frac{2(2-3 \alpha)}{\alpha^{2}\left(\theta^{2}+2\right)}\right] \Gamma\left(\frac{\alpha-2}{\alpha}\right)-\theta^{\frac{2}{\alpha}}\left[1+\frac{(1-3 \alpha)}{\alpha^{2}\left(\theta^{2}+2\right)}\right]^{2} \Gamma^{2}\left(\frac{\alpha-1}{\alpha}\right) .
$$

\subsection{Coefficient of variation of the inverse power Akash distribution}

The ratio of the standard deviation to the mean gives the coefficient of variation (cv) of the inverse power Akash distribution (IPAD) as

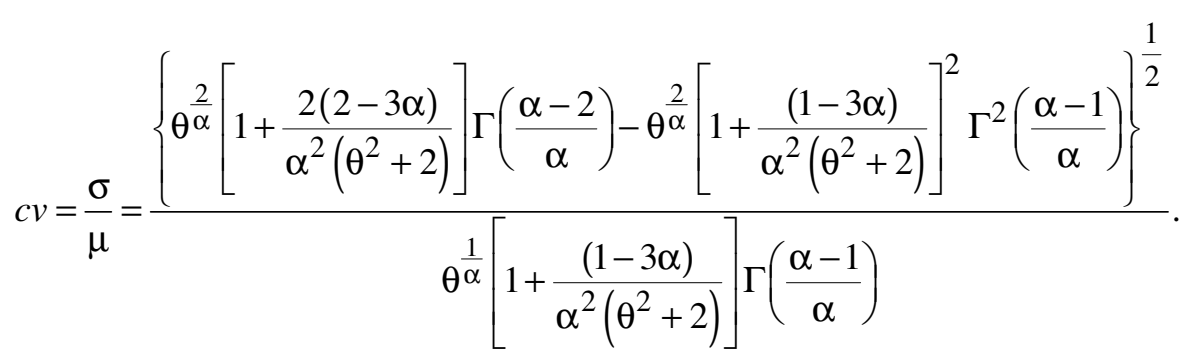




\subsection{Skewness and Kurtosis of the inverse power Akash distribution}

The skewness (sk) of the inverse power Akash distribution (IPAD) is given by

$$
\begin{aligned}
s k(Y) & =\frac{\mu_{3}}{\sigma^{3}} \\
& =\frac{\sum_{j=1}^{3}\left(\begin{array}{l}
3 \\
j
\end{array}\right)\left\{-\theta^{\frac{1}{\alpha}}\left[1+\frac{(1-3 \alpha)}{\alpha^{2}\left(\theta^{2}+2\right)}\right] \Gamma\left(\frac{\alpha-1}{\alpha}\right)\right\}^{3-j} \theta^{\frac{j}{\alpha}}\left[1+\frac{j(j-3 \alpha)}{\alpha^{2}\left(\theta^{2}+2\right)}\right] \Gamma\left(\frac{\alpha-j}{\alpha}\right)}{\left\{\theta^{\frac{2}{\alpha}}\left[1+\frac{2(2-3 \alpha)}{\alpha^{2}\left(\theta^{2}+2\right)}\right] \Gamma\left(\frac{\alpha-2}{\alpha}\right)-\theta^{\frac{2}{\alpha}}\left[1+\frac{(1-3 \alpha)}{\alpha^{2}\left(\theta^{2}+2\right)}\right]^{2} \Gamma^{2}\left(\frac{\alpha-1}{\alpha}\right)\right\}^{3 / 2}}
\end{aligned}
$$

Also, the kurtosis (kur) of the inverse power Akash distribution (IPAD) is

$$
\begin{aligned}
\operatorname{kur}(Y) & =\frac{\mu_{4}}{\sigma^{4}} \\
& =\frac{\sum_{j=1}^{4}\left(\begin{array}{c}
4 \\
j
\end{array}\right)\left\{-\theta^{\frac{1}{\alpha}}\left[1+\frac{(1-3 \alpha)}{\alpha^{2}\left(\theta^{2}+2\right)}\right] \Gamma\left(\frac{\alpha-1}{\alpha}\right)\right\}^{4-j} \theta^{\frac{j}{\alpha}}\left[1+\frac{j(j-3 \alpha)}{\alpha^{2}\left(\theta^{2}+2\right)}\right] \Gamma\left(\frac{\alpha-j}{\alpha}\right)}{\left\{\theta^{\frac{2}{\alpha}}\left[1+\frac{2(2-3 \alpha)}{\alpha^{2}\left(\theta^{2}+2\right)}\right] \Gamma\left(\frac{\alpha-2}{\alpha}\right)-\theta^{\frac{2}{\alpha}}\left[1+\frac{(1-3 \alpha)}{\alpha^{2}\left(\theta^{2}+2\right)}\right]^{2} \Gamma^{2}\left(\frac{\alpha-1}{\alpha}\right)\right\}^{2}}
\end{aligned}
$$

\subsection{Moments generating function of the inverse power Akash distribution}

In addition to moments, many of the interesting features of a statistical distribution can also be obtained through its moment generating function (mgf). Let $Y$ denote a random variable having the inverse power Akash distribution (IPAD) with parameters $\theta$ and $\alpha$, then its moment generating function (mgf) is

$$
M_{Y}(t)=E\left(e^{t Y}\right)=\frac{\alpha \theta^{3}}{\theta^{2}+2} \int_{0}^{\infty} e^{t y}\left(1+y^{-2 \alpha}\right) y^{-\alpha-1} e^{-\theta y^{-\alpha}} d y
$$


Using the series expansion $e^{t y}=\sum_{r=0}^{\infty} \frac{(t y)^{r}}{r !}$ together with $y=x^{-\frac{1}{\alpha}}, \quad x=y^{-\alpha}$ and $\frac{d x}{d y}=-\alpha y^{-\alpha-1}$, the expression in (30) becomes

$$
\begin{aligned}
M_{Y}(t) & =\frac{\theta^{3}}{\theta^{2}+2} \sum_{r=0}^{\infty} \frac{t^{r}}{r !}\left[\int_{0}^{\infty} \frac{e^{-\frac{\theta}{x}}}{4-\frac{r}{\alpha}} d x+\int_{0}^{\infty} \frac{e^{-\frac{\theta}{x}}}{2-\frac{r}{\alpha}} d x\right] \\
& =\frac{\theta^{3}}{\theta^{2}+2} \sum_{r=0}^{\infty} \frac{t^{r}}{r !}\left[\frac{\Gamma\left(3-\frac{r}{\alpha}\right)}{\left.\theta^{3-\frac{r}{\alpha}}+\frac{\Gamma\left(1-\frac{r}{\alpha}\right)}{\theta^{3-\frac{r}{\alpha}}}\right]}\right. \\
& =\sum_{r=0}^{\infty} \frac{t^{r}}{r !} \theta^{\frac{r}{\alpha}}\left[1+\frac{r(r-3 \alpha)}{\alpha^{2}\left(\theta^{2}+2\right)}\right] \Gamma\left(\frac{\alpha-r}{\alpha}\right), \quad \alpha>r .
\end{aligned}
$$

\section{Reliability Analyses}

In this section, we present the survival, hazard rate, reversed hazard, cumulative hazard and odds functions of the inverse power Akash distribution, which are useful in reliability analysis.

\subsection{Survival function for the inverse power Akash distribution}

Let $Y$ be a continuous random variable having the inverse power Akash distribution with parameters $\theta$ and $\alpha$, then the survival function of $Y$ is defined to be

$$
S(y)=1-F(y)=1-\left(1+\frac{\theta y^{-\alpha}\left(\theta y^{-\alpha}+2\right)}{\theta^{2}+2}\right) e^{-\theta y^{-\alpha}} .
$$

The survival function is also known as the reliability function and it indicates the probability of surviving an age $y$ or becoming older than $y$. The study of $S(y)$ is at the heart of survival analysis and reliability theory. It is important in describing systems of components, that is, in calculating systems' reliability.

\subsection{Hazard rate function of the inverse power Akash distribution}

The hazard rate function of a statistical distribution is obtained mathematically as the 
ratio of the probability density function $f(y)$ to the survival function $S(y)$. Thus, the hazard rate function for the inverse power Akash distribution is defined as

$$
\begin{aligned}
h(y) & =\frac{f(y)}{S(y)}=\frac{\frac{\alpha \theta^{3}}{\theta^{2}+2}\left(1+y^{-2 \alpha}\right) y^{-\alpha-1} e^{-\theta y^{-\alpha}}}{1-\left(1+\frac{\theta y^{-\alpha}\left(\theta y^{-\alpha}+2\right)}{\theta^{2}+2}\right) e^{-\theta y^{-\alpha}}} \\
& =\frac{\alpha \theta^{3}\left(1+y^{2 \alpha}\right)}{y^{\alpha+1}\left[-\theta\left(\theta+2 y^{2 \alpha}\right)+\left(\theta^{2}+2\right) y^{2 \alpha}\left(e^{\theta / y^{\alpha}}-1\right)\right]} .
\end{aligned}
$$

For the inverse power Akash distribution (IPAD), the behaviour of $S(y)$ and $h(y)$ for different values of $\theta$ and $\alpha$ are shown in Figures 3 and 4 respectively. The hazard rate function appears always unimodal. Its shape becomes more peaked with increasing values of $\alpha$ and less peaked with increasing values of $\theta$.

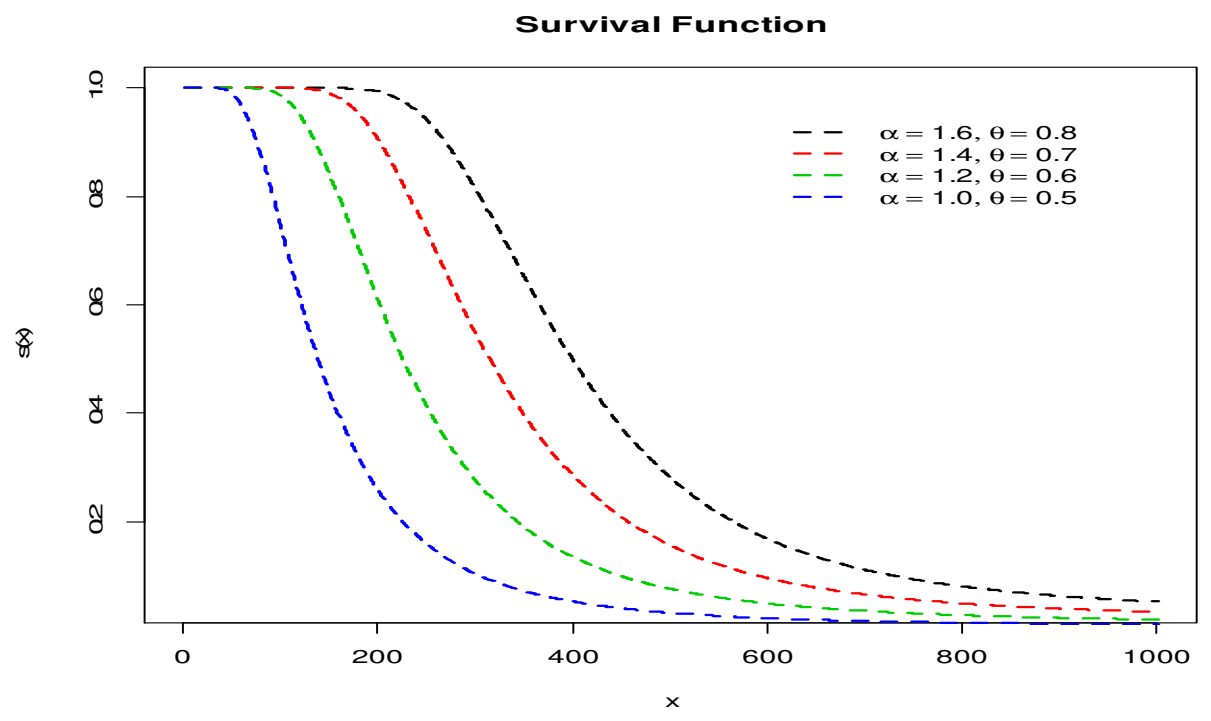

Figure 3. Survival function of the inverse power Akash distribution $\theta$ and $\alpha$. 
Hazard Rate Function

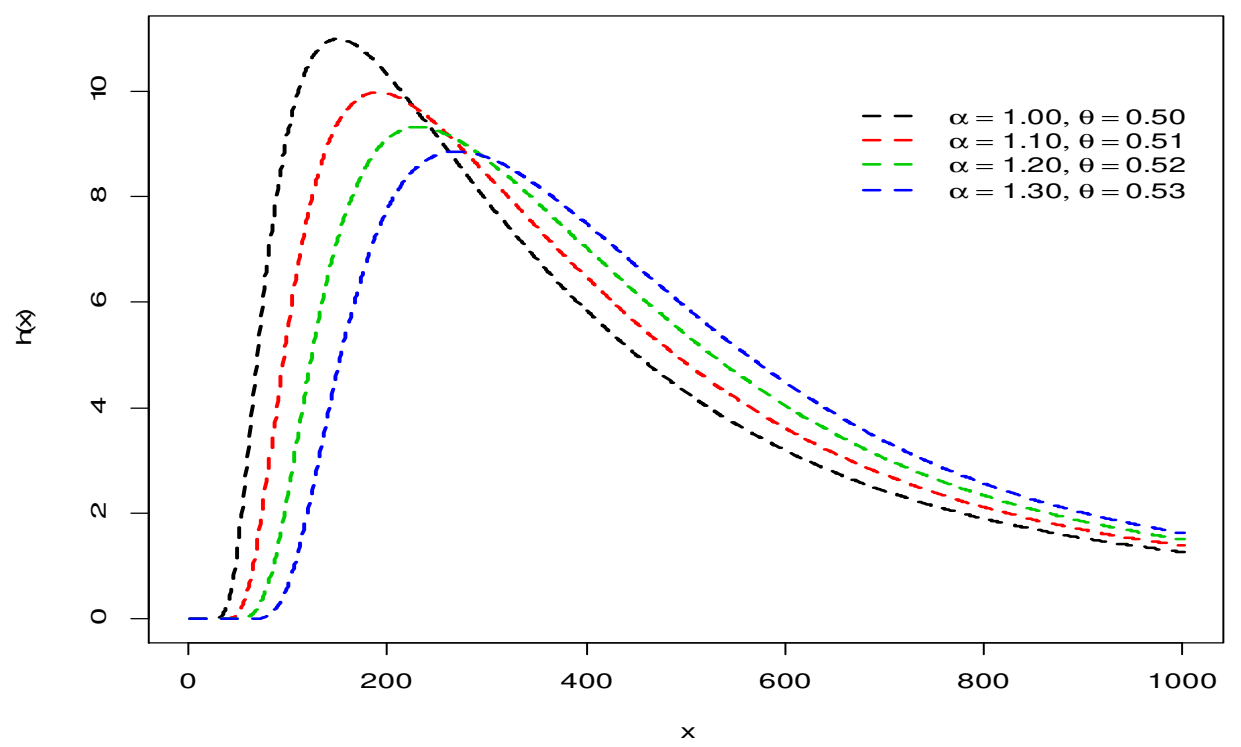

Figure 4. Hazard function of the inverse power Akash distribution $\theta$ and $\alpha$.

\subsection{Reversed hazard rate for the inverse power Akash distribution}

The reversed hazard rate refers to the ratio of the probability density function (pdf) to the cumulative distribution function (cdf). It extends the concept of hazard rate to a reverse time direction and is given by

$$
h_{R}(y)=\frac{f(y)}{F(y)}=\left(\frac{\alpha \theta^{3}}{\theta^{2}+2}\left(1+y^{-2 \alpha}\right) y^{-\alpha-1}\right)\left(1+\frac{\theta y^{-\alpha}\left(\theta y^{-\alpha}+2\right)}{\theta^{2}+2}\right)^{-1} .
$$

The reversed hazard $h_{R}(y)$ describes the probability of an immediate past failure, given that the unit has already failed at time $y$, described by $h(y)$.

\subsection{Cumulative hazard rate function for the inverse power Akash distribution}

The cumulative hazard rate (chr) of the probability density function (pdf) is defined as

$$
H(y)=-\ln [1-F(y)]=-\ln \left\{1-\left(1+\frac{\theta y^{-\alpha}\left(\theta y^{-\alpha}+2\right)}{\theta^{2}+2}\right) e^{-\theta y^{-\alpha}}\right\}
$$


Notably $H(y)$ does not have a probabilistic connotation although it plays a key role in reliability and survivals analysis since $P(Y>y)=e^{-H(y)}, y \geq 0$.

\subsection{Odds function for the inverse power Akash distribution}

The odds function of the inverse power Akash distribution is defined as

$$
\begin{aligned}
O(y) & =\frac{F(y)}{1-F(y)} \\
& =\left[\left(1+\frac{\theta y^{-\alpha}\left(\theta y^{-\alpha}+2\right)}{\theta^{2}+2}\right) e^{-\theta y^{-\alpha}}\right]\left[1-\left(1+\frac{\theta y^{-\alpha}\left(\theta y^{-\alpha}+2\right)}{\theta^{2}+2}\right) e^{-\theta y^{-\alpha}}\right]^{-1} .
\end{aligned}
$$

\section{Rényi Entropy of the Inverse Power Akash Distribution}

Entropy provides tool for quantifying the amount of information (or uncertainty) contained in a random sample regarding its parent population. A large value of entropy implies that there is greater uncertainty in the data. The concept of entropy is important in different areas such as physics, probability and statistics, communication theory, economics and so on. The Rényi, Shannon and Tsallis entropy, among others, are some different forms of entropy. In this paper, the widely used Rényi entropy was considered. Consequently, the Rényi entropy is defined by [12] as

$$
\begin{aligned}
\operatorname{Re}(\eta) & =\frac{1}{1-\eta} \log \left\{\int_{y} f^{\eta}(y) d y\right\}, \quad \eta>0 \text { and } \eta \neq 0 \\
& =\frac{1}{1-\eta} \log \left\{\int_{0}^{\infty}\left[\frac{\alpha \theta^{3}}{\theta^{2}+2}\left(1+y^{-2 \alpha}\right) y^{-\alpha-1} e^{-\theta y^{-\alpha}}\right]^{\eta} d y\right\} \\
& =\frac{1}{1-\eta} \log \left\{\left(\frac{\alpha \theta^{3}}{\theta^{2}+2}\right)^{\eta} \int_{0}^{\infty}\left(1+y^{2 \alpha}\right)^{\eta} y^{-3 \eta \alpha-\eta} e^{-\eta \theta y^{-\alpha}} d y\right\} .
\end{aligned}
$$

Using the fact that $\left(1+y^{2 \alpha}\right)^{\eta}=\sum_{j=0}^{\infty}\left(\begin{array}{l}\eta \\ j\end{array}\right) y^{2 \alpha j}$, one gets 


$$
\operatorname{Re}(\eta)=\frac{1}{1-\eta} \log \left\{\left(\frac{\alpha \theta^{3}}{\theta^{2}+2}\right)^{\eta} \sum_{j=0}^{\infty}\left(\begin{array}{l}
\eta \\
j
\end{array}\right) \int_{0}^{\infty} \frac{e^{-\eta \theta y^{-\alpha}}}{y^{\eta(3 \alpha-1)-2 \alpha j}} d y\right\}
$$

Using $y=x^{-\frac{1}{\alpha}}, x=y^{-\alpha}$ and $\frac{d x}{d y}=-\alpha y^{-\alpha-1}$ and simplifying, we obtain

$$
\begin{aligned}
\operatorname{Re}(\eta)=\frac{1}{1-\eta} \log \left\{\left(\frac{\alpha \theta^{3}}{\theta^{2}+2}\right)^{\eta} \frac{1}{\alpha(\eta \theta)^{\eta / \alpha(3 \alpha-1)-1 / \alpha}}\right. \\
\left.\qquad \sum_{j=0}^{\infty}\left(\begin{array}{l}
\eta \\
j
\end{array}\right)(\eta \theta)^{2 j} \Gamma[\eta / \alpha(3 \alpha-1)-2 j-1 / \alpha]\right\}
\end{aligned}
$$

\section{Stochastic Ordering of the Inverse Power Akash Distribution}

Stochastic ordering of positive continuous random variables is an important tool for judging the comparative behaviour of random variables. The different types of stochastic orderings which are useful in ordering random variables include the usual stochastic order, the hazard rate order, the mean residual life order, and the likelihood ratio order for the random variables under a restricted parameter space. Suppose $X$ and $Y$ are independent random variables with cumulative distribution functions $F_{X}(y)$ and $F_{Y}(y)$ respectively, then $Y$ is said to be smaller than the random variable $X$ in the

1. stochastic order $\left(Y \leq{ }_{s t} X\right)$ if $F_{Y}(y) \geq F_{X}(y)$, for all $y$

2. hazard rate order $\left(Y \leq{ }_{h r} X\right)$ if $h_{Y}(y) \geq h_{X}(y)$, for all $y$

3. mean residual life order $\left(Y \leq{ }_{m r l} X\right)$ if $m_{Y}(y) \geq m_{X}(y)$ for all $y$

4. likelihood ratio order $\left(Y \leq{ }_{l r} X\right)$ if $\frac{f_{Y}(y)}{f_{X}(y)}$ decreases in $y$.

The inverse power Akash distribution is ordered with respect to the strongest "likelihood ratio" ordering. To show the flexibility of the IPAD, its likelihood ratio is defined as 


$$
\begin{aligned}
\frac{f_{Y}(y)}{f_{X}(y)}= & \frac{\frac{\alpha_{1} \theta_{1}^{3}}{\theta_{1}^{2}+2}\left(1+y^{-2 \alpha_{1}}\right) y^{-\alpha_{1}-1} e^{-\theta_{1} y^{-\alpha_{1}}}}{\frac{\alpha_{2} \theta_{2}^{3}}{\theta_{2}^{2}+2}\left(1+y^{-2 \alpha_{2}}\right) y^{-\alpha_{2}-1} e^{-\theta_{2} y^{-\alpha_{2}}}} \\
& =\left(\frac{\alpha_{1} \theta_{1}^{3}}{\alpha_{2} \theta_{2}^{3}}\right)\left(\frac{\theta_{2}^{2}+2}{\theta_{1}^{2}+2}\right) \frac{\left(1+y^{2 \alpha_{1}}\right)}{\left(1+y^{2 \alpha_{2}}\right)} y^{3\left(\alpha_{2}-\alpha_{1}\right)} e^{\theta_{2} y^{-\alpha_{2}}-\theta_{1} y^{-\alpha_{1}}} .
\end{aligned}
$$

Next, the log of the likelihood ratio in (39) is

$$
\begin{aligned}
\ln \left(\frac{f_{Y}(y)}{f_{X}(y)}\right)= & \ln \left(\frac{\alpha_{1} \theta_{1}^{3}}{\alpha_{2} \theta_{2}^{3}}\right)+\ln \left(\frac{\theta_{2}^{2}+2}{\theta_{1}^{2}+2}\right)+\ln \left(1+y^{2 \alpha_{1}}\right) \\
& -\ln \left(1+y^{2 \alpha_{2}}\right)+3\left(\alpha_{2}-\alpha_{2}\right) \ln y+\theta_{2} y^{-\alpha_{2}}-\theta_{1} y^{-\alpha_{1}} .
\end{aligned}
$$

Differentiating (40) with respect to $y$, we obtain

$$
\begin{aligned}
\frac{d}{d y} \ln \left(\frac{f_{Y}(y)}{f_{X}(y)}\right)= & \frac{2 \alpha_{1} y^{2 \alpha_{1}-1}}{1+y^{2 \alpha_{1}}}-\frac{2 \alpha_{2} y^{2 \alpha_{2}-1}}{1+y^{2 \alpha_{2}}}+\frac{3\left(\alpha_{2}-\alpha_{1}\right)}{y} \\
& +\alpha_{2} \theta_{2} y^{-\left(\alpha_{2}+1\right)}-\alpha_{1} \theta_{1} y^{-\left(\alpha_{1}+1\right)}
\end{aligned}
$$

If $\alpha_{1}=\alpha_{2}=\alpha$ and $\theta_{1} \geq \theta_{1}$, then (40) and (41) respectively reduces to

$$
\frac{f_{Y}(y)}{f_{X}(y)}=\left(\frac{\theta_{1}^{3}}{\theta_{2}^{3}}\right)\left(\frac{\theta_{2}^{2}+2}{\theta_{1}^{2}+2}\right) e^{\left(\theta_{2}-\theta_{1}\right) / y^{\alpha}}
$$

and

$$
\frac{d}{d y} \ln \left(\frac{f_{Y}(y)}{f_{X}(y)}\right)=\frac{\alpha\left(\theta_{2}+\theta_{1}\right)}{y^{\alpha+1}}
$$

which is decreasing in $y$ for $\theta_{2} \geq \theta_{1}$ or (or for $\theta_{1}=\theta_{2}$ ). The results in (42) and (43) clearly show that if $Y \sim\left(\theta_{1}, \alpha_{1}\right)$ and $X \sim\left(\theta_{2}, \alpha_{2}\right)$, then $Y \geq l_{r} X$ and hence, $Y \geq_{h r} X$, $Y \geq_{m r l} X$ and $Y \geq_{s t} X$ provided $\theta_{2} \geq \theta_{1}$ or (or for $\theta_{1}=\theta_{2}$ ). 


\section{Maximum Likelihood Estimators of the Inverse Power Akash Distribution}

In estimation theory, the method of maximum likelihood have been used more often to find the parameters of statistical distributions due to the fact that it possesses the consistency, asymptotic efficiency and invariance properties. Thus, to obtain the maximum likelihood estimators of parameters of the inverse power Akash distribution, let $Y_{1}, Y_{2}, \ldots, Y_{n}$ constitute a random sample of size $n$ from this distribution and define the likelihood function of the random sample as

$$
L(\theta, \alpha)=\prod_{i=1}^{n} \frac{\alpha \theta^{3}}{\theta^{2}+2}\left(1+y_{i}^{-2 \alpha}\right) y_{i}^{-\alpha-1} e^{-\theta y_{i}^{-\alpha}}=\left(\frac{\alpha \theta^{3}}{\theta^{2}+2}\right)^{n} e^{-\theta \sum_{i=1}^{n} y_{i}^{-\alpha}} \prod_{i=1}^{n} \frac{\left(1+y_{i}^{2 \alpha}\right)}{y_{i}^{3 \alpha+1}} .
$$

Taking the natural $\log$ of (44), one obtains the log-likelihood function of the random sample as

$$
\begin{aligned}
\ln L(\theta, \alpha)= & n\left[\ln (\alpha)+3 \ln (\theta)-\ln \left(\theta^{2}+2\right)\right] \\
& -\theta \sum_{i=1}^{n} y_{i}^{-\alpha}+\sum_{i=1}^{n} \ln \left(1+y_{i}^{2 \alpha}\right)-(3 \alpha+1) \sum_{i=1}^{n} \ln y_{i} .
\end{aligned}
$$

Taking the partial derivatives of (45) with respect $\theta$ and $\alpha$ respectively leads to

$$
\begin{aligned}
& \frac{\partial \ln L(\theta, \alpha)}{\partial \alpha}=\frac{n}{\alpha}+\theta \sum_{i=1}^{n} y_{i}^{-\alpha} \ln y_{i}+2 \sum_{i=1}^{n} \frac{y_{i}^{2 \alpha} \ln y_{i}}{1+y_{i}^{2 \alpha}}-3 \sum_{i=1}^{n} \ln y_{i} \\
& \frac{\partial \ln L(\theta, \alpha)}{\partial \theta}=\frac{3 n}{\theta}-\frac{2 n \theta}{\theta^{2}+2}-\sum_{i=1}^{n} y_{i}^{-\alpha} .
\end{aligned}
$$

The maximum likelihood estimates $(\hat{\alpha}$ and $\hat{\theta})$ of parameters $(\alpha$ and $\theta)$ are obtained by solving these nonlinear systems of equations $\partial \ln L(\theta, \alpha) / \partial \alpha=0$ and $\partial \ln L(\theta, \alpha) / \partial \theta$ $=0$. Consequently,

$$
\frac{n}{\alpha}+\theta \sum_{i=1}^{n} y_{i}^{-\alpha} \ln y_{i}+2 \sum_{i=1}^{n} \frac{y_{i}^{2 \alpha} \ln y_{i}}{1+y_{i}^{2 \alpha}}-3 \sum_{i=1}^{n} \ln y_{i}=0
$$




$$
\frac{3 n}{\theta}-\frac{2 n \theta}{\theta^{2}+2}-\sum_{i=1}^{n} y_{i}^{-\alpha}=0 .
$$

The maximum likelihood estimates of $\alpha$ and $\theta$ can is obtained by solving the nonlinear system of equations (48) and (49). It is usually more convenient to use nonlinear optimization algorithms such as quasi-Newton algorithm to numerically maximize the log-likelihood function. The $\mathrm{R}$ package provides nonlinear optimization for solving such problems.

\section{The Asymptotic Distribution and Approximate Confidence Interval Estimators of $(\hat{\alpha}, \hat{\theta})$ for the Inverse Power Akash Distribution}

It is clear that the log-likelihood equations defined in (48) and (49) does not yield closed-form solution. As a result, the estimates of the unknown parameters $\alpha$ and $\theta$ obtained in this work are approximate solution. In what follows, their corresponding confidence intervals cannot be constructed explicitly. Thus, there is need to find the approximate confidence intervals of $\alpha$ and $\theta$. To do this, one is required to obtain the asymptotic distribution of the maximum likelihood estimators of $\alpha$ and $\theta$. In this regard, we first obtain the second-order partial derivatives, which are required in order to determine the Fisher information matrix. Thus,

$$
\begin{aligned}
& \frac{\partial^{2} \ln L(\theta, \alpha)}{\partial \theta^{2}}=-\frac{3 n}{\theta}+\frac{2 n\left(\theta^{2}-2\right)}{\theta^{2}+2} \\
& \frac{\partial^{2} \ln L(\theta, \alpha)}{\partial \alpha^{2}}=-\frac{n}{\alpha^{2}}-\theta \sum_{i=1}^{n} y_{i}^{-\alpha}\left(\ln y_{i}\right)^{2}+4 \sum_{i=1}^{n} \frac{y_{i}^{2 \alpha}\left(\ln y_{i}\right)^{2}}{\left(1+y_{i}^{2 \alpha}\right)^{2}} \\
& \frac{\partial^{2} \ln L(\theta, \alpha)}{\partial \theta \partial \alpha}=\sum_{i=1}^{n} y_{i}^{-\alpha} \ln y_{i} .
\end{aligned}
$$

Now, we take expectation of (50) to get

$$
E\left[\frac{\partial^{2} \ln L(\theta, \alpha)}{\partial \theta^{2}}\right]=-\frac{3 n}{\theta}+\frac{2 n\left(\theta^{2}-2\right)}{\theta^{2}+2} .
$$


Similarly, the expectations of (51) is given by

$$
E\left[\frac{\partial^{2} \ln L(\theta, \alpha)}{\partial \alpha^{2}}\right]=-\frac{n}{\alpha^{2}}-\theta \sum_{i=1}^{n} E\left[Y_{i}^{-\alpha}\left(\ln y_{i}\right)^{2}\right]+4 \sum_{i=1}^{n} E\left[\frac{Y_{i}^{2 \alpha}\left(\ln Y_{i}\right)^{2}}{\left(1+Y_{i}^{2 \alpha}\right)^{2}}\right] .
$$

To solve (54), one is required to find the expressions for $E\left[Y_{i}^{-\alpha}\left(\ln Y_{i}\right)^{2}\right]$ and $E\left[\frac{Y_{i}^{2 \alpha}\left(\ln Y_{i}\right)^{2}}{\left(1+Y_{i}^{2 \alpha}\right)^{2}}\right]$ respectively. Thus,

$$
\begin{aligned}
E\left[Y^{-\alpha}(\ln Y)^{2}\right] & =\frac{\alpha \theta^{3}}{\theta^{2}+2} \int_{0}^{\infty} y^{-\alpha}(\ln y)^{2}\left(1+y^{-2 \alpha}\right) y^{-\alpha-1} e^{-\theta y^{-\alpha}} d y \\
& =\frac{\alpha \theta^{3}}{\theta^{2}+2} \int_{0}^{\infty} y_{i}^{-\alpha}\left(\ln y_{i}\right)^{2} \frac{1+y^{2 \alpha}}{y^{3 \alpha+1}} e^{-\theta y^{-\alpha}} d y
\end{aligned}
$$

Letting $y=x^{-\frac{1}{\alpha}}, y^{-\alpha}=x, y^{2 \alpha}=x^{-2}, y^{3 \alpha+1}=x^{-(2+1 / \alpha)}, d y=-\frac{1}{\alpha} x^{-\frac{1}{\alpha}-1} d x$ in (54), one obtains

$$
\begin{aligned}
E\left[Y^{-\alpha}(\ln Y)^{2}\right] & =\frac{\alpha \theta^{3}}{\theta^{2}+2} \int_{0}^{\infty} x\left(\ln x^{-\frac{1}{\alpha}}\right)^{2} \frac{1+x^{-2}}{x^{-(3+1 / \alpha)}} e^{-\theta x}\left(-\frac{1}{\alpha} x^{-\frac{1}{\alpha}-1} d x\right) \\
& =\frac{\theta^{3}}{\alpha^{2}\left(\theta^{2}+2\right)} \int_{0}^{\infty} \ln x\left(x+x^{3}\right) e^{-\theta x} d x \\
& =\frac{\theta^{3}}{\alpha^{2}\left(\theta^{2}+2\right)}\left[\int_{0}^{\infty} x^{2-1} e^{-\theta x}(\ln x)^{2} d x+\int_{0}^{\infty} x^{4-1} e^{-\theta x}(\ln x)^{2} d x\right] .
\end{aligned}
$$

To resolve the integrals in (56), we recall from [13] that

$$
\int_{0}^{\infty} x^{\nu-1} e^{-\mu x}(\ln x)^{2} d x=\frac{\Gamma(v)}{\mu^{v}}\left\{[\psi(v)-\ln \mu]^{2}+\zeta(2, v)\right\}, \quad v, \mu>0
$$


and

$$
\zeta(z, v)=\sum_{m=0}^{\infty} \frac{1}{(v+m)^{2}}, \quad z>1, \quad v \neq 0,-1,-2, \ldots
$$

where $\Gamma(v)$ is the gamma function of $v, \psi(v)$ is the digamma function of $v$, and $\zeta(z, v)$ is the Riemann's zeta function of $(z, v)$. Using (57) and (58) in (56), we have

$$
\begin{aligned}
& E\left[Y^{-\alpha}(\ln Y)^{2}\right] \\
= & \frac{\theta^{3}}{\alpha^{2}\left(\theta^{2}+2\right)}\left\{\frac{\Gamma(2)}{\theta^{2}}\left\{[\psi(3)-\ln \theta]^{2}+\zeta(2,2)\right\}+\frac{\Gamma(4)}{\theta^{4}}\left\{[\psi(3)-\ln \theta]^{2}+\zeta(2,4)\right\}\right\} \\
= & \frac{1}{\alpha^{2} \theta^{2}\left(\theta^{2}+2\right)}\left\{\theta^{2}\left[(\psi(2)-\ln \theta)^{2}+\zeta(2,2)\right]+6\left[(\psi(4)-\ln \theta)^{2}+\zeta(2,4)\right]\right\} .
\end{aligned}
$$

Again, we obtain the following expectation

$$
\begin{aligned}
E\left[\frac{Y^{2 \alpha}(\ln Y)^{2}}{\left(1+Y^{2 \alpha}\right)^{2}}\right] & =\frac{\alpha \theta^{3}}{\theta^{2}+2} \int_{0}^{\infty} \frac{y_{i}^{2 \alpha}\left(\ln y_{i}\right)^{2}}{\left(1+y_{i}^{2 \alpha}\right)^{2}}\left(1+y^{-2 \alpha}\right) y^{-\alpha-1} e^{-\theta y^{-\alpha}} d y \\
& =\frac{\alpha \theta^{3}}{\theta^{2}+2} \int_{0}^{\infty} \frac{y^{2 \alpha}\left(\ln y_{i}\right)^{2}}{\left(1+y^{2 \alpha}\right)^{2}} \frac{1+y^{2 \alpha}}{y^{3 \alpha+1}} e^{-\theta y^{-\alpha}} d y .
\end{aligned}
$$

Putting $y=x^{-\frac{1}{\alpha}}, y^{-\alpha}=x, y^{2 \alpha}=x^{-2}, y^{3 \alpha+1}=x^{-(2+1 / \alpha)}, d y=-\frac{1}{\alpha} x^{-\frac{1}{\alpha}-1} d x$ into (60), we have

$$
\begin{aligned}
E\left[\frac{Y^{2 \alpha}(\ln Y)^{2}}{\left(1+Y^{2 \alpha}\right)^{2}}\right] & =\frac{\alpha \theta^{3}}{\theta^{2}+2} \int_{0}^{\infty} \frac{x^{-2}\left(\ln x^{-\frac{1}{\alpha}}\right)^{2}}{\left(1+x^{-2}\right)^{2}} \frac{1+x^{-2}}{x^{-(3+1 / \alpha)}} e^{-\theta x}\left(-\frac{1}{\alpha} x^{-\frac{1}{\alpha}-1} d x\right) \\
& =\frac{\theta^{3}}{\alpha^{2}\left(\theta^{2}+2\right)} \int_{0}^{\infty} \frac{x^{-2}\left(1+x^{2}\right)}{\left(1+x^{-2}\right)^{2}}(\ln x)^{2} e^{-\theta x} d x
\end{aligned}
$$




$$
\begin{aligned}
& =\frac{\theta^{3}}{\alpha^{2}\left(\theta^{2}+2\right)} \int_{0}^{\infty} \frac{\left(1+x^{-2}\right)}{\left(1+x^{-2}\right)^{2}}(\ln x)^{2} e^{-\theta x} d x \\
& =\frac{\theta^{3}}{\alpha^{2}\left(\theta^{2}+2\right)} \int_{0}^{\infty} \frac{x^{2}}{\left(1+x^{2}\right)}(\ln x)^{2} e^{-\theta x} d x \\
& =\frac{\theta^{3}}{\alpha^{2}\left(\theta^{2}+2\right)} \int_{0}^{\infty}\left(1-\frac{1}{1+x^{2}}\right)(\ln x)^{2} e^{-\theta x} d x \\
& =\frac{\theta^{3}}{\alpha^{2}\left(\theta^{2}+2\right)}\left[\int_{0}^{\infty} x^{1-1} e^{-\theta x}(\ln x)^{2} d x-\int_{0}^{\infty} \frac{(\ln x)^{2}}{1+x^{2}} e^{-\theta x} d x\right] \\
& =\frac{\theta^{2}}{\alpha^{2}\left(\theta^{2}+2\right)}\left[\left\{[\psi(1)-\ln \theta]^{2}+\zeta(2,1)\right\}-\theta J(\theta)\right]
\end{aligned}
$$

where $J(\theta)=\int_{0}^{\infty} \frac{(\ln x)^{2}}{1+x^{2}} e^{-\theta x} d x$.

Substituting (59) and (61) into (54), we obtain

$$
\begin{aligned}
& E\left[\frac{\partial^{2} \ln L(\theta, \alpha)}{\partial \alpha^{2}}\right] \\
= & -\frac{n}{\alpha^{2}}-\frac{n \theta}{\alpha^{2} \theta^{2}\left(\theta^{2}+2\right)}\left\{\theta^{2}\left[(\psi(2)-\ln \theta)^{2}+\zeta(2,2)\right]+6\left[(\psi(4)-\ln \theta)^{2}+\zeta(2,4)\right]\right\} \\
& +\frac{4 n \theta^{2}}{\alpha^{2}\left(\theta^{2}+2\right)}\left[\theta\left\{[\psi(1)-\ln \theta]^{2}+\zeta(2,1)\right\}+J(\theta)\right] .
\end{aligned}
$$

Finally, we obtain the expectation

$$
E\left[\frac{\partial^{2} \ln L(\theta, \alpha)}{\partial \theta \partial \alpha}\right]=\sum_{i=1}^{n} E\left[Y_{i}^{-\alpha} \ln Y_{i}\right] .
$$


But

$$
\begin{aligned}
E\left[Y^{-\alpha} \ln Y\right] & =\frac{\alpha \theta^{3}}{\theta^{2}+2} \int_{0}^{\infty} y^{-\alpha} \ln y\left(1+y^{-2 \alpha}\right) y^{-\alpha-1} e^{-\theta y^{-\alpha}} d y \\
& =\frac{n \alpha \theta^{3}}{\theta^{2}+2} \int_{0}^{\infty} y^{-\alpha} \ln y_{i} \frac{1+y^{2 \alpha}}{y^{3 \alpha+1}} e^{-\theta y^{-\alpha}} d y
\end{aligned}
$$

Putting $y=x^{-\frac{1}{\alpha}}, \quad y^{-\alpha}=x, \quad y^{2 \alpha}=x^{-2}, \quad y^{3 \alpha+1}=x^{-(2+1 / \alpha)}, \quad d y=-\frac{1}{\alpha} x^{-\frac{1}{\alpha}-1} d x$ into (64) leads to

$$
\begin{aligned}
E\left[Y^{-\alpha} \ln Y\right] & =\frac{\alpha \theta^{3}}{\theta^{2}+2} \int_{0}^{\infty} x \ln x^{-\frac{1}{\alpha}} \frac{1+x^{-2}}{x^{-(3+1 / \alpha)}} e^{-\theta x}\left(-\frac{1}{\alpha} x^{-\frac{1}{\alpha}-1} d x\right) \\
& =\frac{\theta^{3}}{\alpha\left(\theta^{2}+2\right)} \int_{0}^{\infty} \ln x\left(x+x^{3}\right) e^{-\theta x} d x \\
& =\frac{\theta^{3}}{\alpha\left(\theta^{2}+2\right)}\left[\int_{0}^{\infty} x^{2-1} e^{-\theta x} \ln x d x+\int_{0}^{\infty} x^{4-1} e^{-\theta x} \ln x d x\right] .
\end{aligned}
$$

In [13], $\int_{0}^{\infty} x^{v-1} e^{-\mu x} \ln x d x=\frac{\Gamma(v)}{\mu^{v}}[\psi(v)-\ln \mu], v, \mu>0$. Consequently, (65) becomes

$$
\begin{aligned}
E\left(Y^{-\alpha} \ln Y\right) & =\frac{\theta^{3}}{\alpha\left(\theta^{2}+2\right)}\left\{\frac{\Gamma(2)}{\theta^{2}}[\psi(2)-\ln \theta]+\frac{\Gamma(4)}{\theta^{4}}[\psi(4)-\ln \theta]\right\} \\
& =\frac{1}{\alpha \theta\left(\theta^{2}+2\right)}\left\{\theta^{2}[\psi(2)-\ln \theta]+6[\psi(4)-\ln \theta]\right\} .
\end{aligned}
$$

Substituting (66) into (63), one obtains

$$
E\left[\frac{\partial^{2} \ln L(\theta, \alpha)}{\partial \theta \partial \alpha}\right]=\frac{n}{\alpha \theta\left(\theta^{2}+2\right)}\left\{\theta^{2}[\psi(2)-\ln \theta]+6[\psi(4)-\ln \theta]\right\} .
$$

The expressions defined in (53), (62) and (66) are used to find the Fisher information 
matrix $I(\theta, \alpha)$ for the IPAD. However, the elements of the Fisher information matrix are given respectively as

$$
\begin{aligned}
I_{11} & =-E\left[\frac{\partial^{2} \ln L(\theta, \alpha)}{\partial \theta^{2}}\right]=\frac{3}{\theta}-\frac{2\left(\theta^{2}-2\right)}{\theta^{2}+2} \\
I_{22} & =-E\left[\frac{\partial^{2} \ln L(\theta, \alpha)}{\partial \alpha^{2}}\right] \\
& =\frac{1}{\alpha^{2}}+\frac{1}{\alpha^{2} \theta\left(\theta^{2}+2\right)}\left\{\theta^{2}\left[(\psi(2)-\ln \theta)^{2}+\zeta(2,2)\right]+6\left[(\psi(4)-\ln \theta)^{2}+\zeta(2,4)\right]\right. \\
I_{12} & \left.=I_{21}=-E\left[\frac{\partial^{2} \ln L(\theta, \alpha)}{\partial \theta \partial \alpha}\right]\left[(\psi(1)-\ln \theta)^{2}+\zeta(2,1)\right]+4 \theta^{3} J(\theta)\right\} \\
& =\frac{-\theta^{2}\left[(\psi(2)-\ln \theta)^{2}+\zeta(2,2)\right]-6\left[(\psi(4)-\ln \theta)^{2}+\zeta(2,4)\right]}{\alpha^{2} \theta^{2}\left(\theta^{2}+2\right)} .
\end{aligned}
$$

According to [14], the asymptotic distribution of $(\hat{\alpha}, \hat{\beta})$ as $n \rightarrow \infty$ is the bivariate normal distribution with means $(\alpha, \beta)$ and variance-covariance matrix $n^{-1} I_{F}^{-1}(\hat{\alpha}, \hat{\beta})$, provided certain regularity conditions are met. In line with the work by [14], the asymptotic variance of the maximum likelihood estimates $\hat{\theta}$ and $\hat{\alpha}$ are respectively given by

$$
\operatorname{Var}(\hat{\theta})=n^{-1} \frac{I_{11}}{I_{11} I_{22}-I_{12}^{2}}
$$

and

$$
\operatorname{Var}(\hat{\alpha})=n^{-1} \frac{I_{22}}{I_{11} I_{22}-I_{11}^{2}} .
$$

Also, the asymptotic coverage lengths of $\hat{\alpha}$ and $\hat{\theta}$ with significance level $\gamma$ are respectively 


$$
2 z_{\gamma / 2} \sqrt{\frac{I_{11}}{n\left(I_{11} I_{22}-I_{12}^{2}\right)}}
$$

and

$$
2 z_{\gamma / 2} \sqrt{\frac{I_{22}}{n\left(I_{11} I_{22}-I_{12}^{2}\right)}},
$$

where $z_{\gamma / 2}$ is the quantile of the standard normal distribution.

In practice, the distribution of $(\hat{\alpha}-\alpha, \hat{\theta}-\theta)$ can be approximated by a bivariate normal distribution with zero means and covariance matrix

$$
\begin{aligned}
n^{-1} I_{F}^{-1}(\hat{\alpha}, \hat{\theta}) & =n^{-1}\left(\begin{array}{ll}
\hat{I}_{11} & \hat{I}_{12} \\
\hat{I}_{21} & \hat{I}_{22}
\end{array}\right)^{-1} \\
& =\frac{1}{n\left(\hat{I}_{11} \hat{I}_{22}-\hat{I}_{22}\right)}\left(\begin{array}{cc}
\hat{I}_{22} & -\hat{I}_{12} \\
-\hat{I}_{21} & \hat{I}_{11}
\end{array}\right)=n^{-1}\left(\begin{array}{cc}
V_{11} & -V_{12} \\
V_{21} & V_{22}
\end{array}\right)
\end{aligned}
$$

say for $n$ sufficiently large. This approximation can be used to construct confidence intervals and test of hypotheses. For example, $100(1-\gamma)$ confidence intervals for $\theta$ and $\alpha$ are respectively given by

$$
\hat{\theta} \pm z_{\gamma / 2} \sqrt{\frac{V_{11}}{n}}
$$

and

$$
\hat{\alpha} \pm z_{\gamma / 2} \sqrt{\frac{V_{22}}{n}} \text {. }
$$

\section{Distributions of Order Statistics for the Inverse Power Akash Distribution}

Let $Y_{1}, Y_{2}, \ldots, Y_{n}$ be a random sample of size $n$ from the inverse power Akash distribution with cumulative distribution function (cdf) $F(y)$ and probability density function $f(y)$. Then, $Y_{(1)}, Y_{(2)}, \ldots, Y_{(n)}$ denote corresponding order statistics, where $Y_{(1)} \leq Y_{(2)} \leq \ldots \leq Y_{(n)}, \quad Y_{(1)}=\min \left(Y_{1}, Y_{2}, \ldots, Y_{n}\right) \quad$ and $Y_{(n)}=\max \left(Y_{1}, Y_{2}, \ldots, Y_{n}\right)$. The probability density function (pdf) of the $k^{\text {th }}$ order statistic is given by 


$$
\begin{aligned}
& f_{Y_{(k)}}(y)=\frac{n !}{(k-1) !(n-k) !}[F(y)]^{k-1}[1-F(y)]^{n-k} f(y) \\
& =\frac{n !}{(k-1) !(n-k) !} \sum_{l=0}^{n-k}\left(\begin{array}{c}
n-k \\
l
\end{array}\right)(-1)^{l}[F(y)]^{k+l-1} f(y) \\
& =\frac{n ! \alpha \theta^{3}}{(k-1) !(n-k) !\left(\theta^{2}+2\right)} \sum_{l=0}^{n-k}\left(\begin{array}{c}
n-k \\
l
\end{array}\right)(-1)^{l} \\
& \left.\times\left\{1+\frac{\theta y^{-\alpha}\left(\theta y^{-\alpha}+2\right)}{\theta^{2}+2}\right] e^{-\theta y^{-\alpha}}\right\}^{k+l-1}\left(1+y^{-2 \alpha}\right) y^{-\alpha-1} e^{-\theta y^{-\alpha}} \\
& =\frac{n ! \alpha \theta^{3}}{(k-1) !(n-k) !\left(\theta^{2}+2\right)} \sum_{l=0}^{n-k}\left(\begin{array}{c}
n-k \\
l
\end{array}\right)(-1)^{l}\left[1+\frac{\theta y^{-\alpha}\left(\theta y^{-\alpha}+2\right)}{\theta^{2}+2}\right]^{k+l-1} \\
& \times\left(\frac{1+y^{2 \alpha}}{y^{3 \alpha+1}}\right) e^{-(k+l) \theta y^{-\alpha}} .
\end{aligned}
$$

If $k=1$ in (78), one obtains the probability density function (pdf) of the first order statistic $Y_{(1)}$ for the inverse power Akash distribution as

$$
f_{Y_{(1)}}(y)=\frac{n \alpha \theta^{3}}{\left(\theta^{2}+2\right)} \sum_{l=0}^{n-1}\left(\begin{array}{c}
n-1 \\
l
\end{array}\right)(-1)^{l}\left[1+\frac{\theta y^{-\alpha}\left(\theta y^{-\alpha}+2\right)}{\theta^{2}+2}\right]^{l}\left(\frac{1+y^{2 \alpha}}{y^{3 \alpha+1}}\right) e^{-(l+1) \theta y^{-\alpha}} .
$$

Similarly for $k=n$ in (79), we obtain the probability density function (pdf) of the $n$th order statistic $Y_{(n)}$ for the inverse power Akash distribution as

$$
f_{Y_{(n)}}(y)=\frac{n \alpha \theta^{3}}{\left(\theta^{2}+2\right)} \sum_{l=0}^{n-1}\left(\begin{array}{c}
n-1 \\
l
\end{array}\right)(-1)^{l}\left[1+\frac{\theta y^{-\alpha}\left(\theta y^{-\alpha}+2\right)}{\theta^{2}+2}\right]^{n+l-1}\left(\frac{1+y^{2 \alpha}}{y^{3 \alpha+1}}\right) e^{-(n+l) \theta y^{-\alpha}}
$$




\section{Numerical Examples}

In this section, two real-life data sets are used to illustrate the importance and flexibility of the inverse power Akash distribution proposed in this work. The first and second data sets, which appeared in the work [15], are provided in Tables 1 and 2 respectively.

Table 1.Uncensored breaking stress of carbon fibres in (Gba).

0.92, 0.928, 0.997, 0.9971, 1.061, 1.117, 1.162, 1.183, 1.187, 1.192, 1.196, 1.213, 1.215, $1.2199,1.22,1.224,1.225,1.228,1.237,1.24,1.244,1.259,1.261,1.263,1.276,1.31$, $1.321,1.329,1.331,1.337,1.351,1.359,1.388,1.408,1.449,1.4497,1.45,1.459,1.471$, $1.475,1.477,1.48,1.489,1.501,1.507,1.515,1.53,1.5304,1.533,1.544,1.5443,1.552$, 1.556, 1.562, 1.566, 1.585, 1.586, 1.599, 1.602, 1.614, 1.616, 1.617, 1.628, 1.684, 1.711, $1.718,1.733,1.738,1.743,1.759,1.777,1.794,1.799,1.806,1.814,1.816,1.828,1.83$, $1.884,1.892,1.944,1.972,1.984,1.987,2.02,2.0304,2.029,2.035,2.037,2.043,2.046$, 2.059, 2.111, 2.165, 2.686, 2.778, 2.972, 3.504, 3.863, 5.306

Source: Mahmoud and Mandouh [15].

Table 2: Uncensored strengths of glass fibres.

$1.014,1.081,1.082,1.185,1.223,1.248,1.267,1.271,1.272,1.275,1.276,1.278,1.286$, $1.288,1.292,1.304,1.306,1.355,1.361,1.364,1.379,1.409,1.426,1.459,1.46,1.476$, $1.481,1.484,1.501,1.506,1.524,1.526,1.535,1.541,1.568,1.579,1.581,1.591,1.593$, $1.602,1.666,1.67,1.684,1.691,1.704,1.731,1.735,1.747,1.748,1.757,1.800,1.806$, $1.867,1.876,1.878,1.91,1.916,1.972,2.012,2.456,2.592,3.197,4.121$

Source: Mahmoud and Mandouh [15].

The inverse power Akash distribution is fitted the to the two data sets by using the method of maximum likelihood and the results are compared with the other competitive models namely, Akash (A), Inverse Akash (IA) and Power Akash (PA) distributions respectively.

Next, some criteria like the Akaike information criterion (AIC), Bayesian information criterion (BIC), and Consistent Akaike information criterion (CAIC) are used to verify which of the aforementioned distributions fits the research data better. The formulae for computing the vales of AIC, BIC and CAIC are respectively given by

$$
\begin{aligned}
A I C & =2 k-2 l \\
B I C & =k \ln (n)-2 l \\
C A I C & =\frac{2 n k}{n-k-1}-2 l,
\end{aligned}
$$

where $l$ denotes the log-likelihood function evaluated at the maximum likelihood 
estimates, $k$ is the number of model parameters, $n$ is the sample size. For calculation of the analytical measures, the optimum () R-function with the argument method= "BFGS".

A distribution is said to provide the best fit to the data if among all the distributions under consideration, it corresponds to minimum values of AIC, BIC, CAIC and the loglikelihood respectively. The maximum likelihood estimates with the standard error of the fitted models and the corresponding model selection criteria for data sets 1 and 2 are presented in Tables 3 and 4.

Table 3. Estimates of model parameter with standard errors and corresponding values of model selection criteria for the distributions fitted to data set 1

\begin{tabular}{|l|l|l|l|l|l|l|l|l|}
\hline Distribution & $\begin{array}{l}\text { Parameter } \\
\text { Estimates }\end{array}$ & $\begin{array}{l}\text { Standard } \\
\text { Error }\end{array}$ & $\log L$ & AIC & BIC & CAIC & \multicolumn{2}{|c|}{$\begin{array}{l}\text { C5\% } \\
\text { Interval } \\
L\end{array}$} \\
\hline IPAD & $\begin{array}{l}\hat{\theta}=5.0911 \\
\hat{\alpha}=4.5137\end{array}$ & $\begin{array}{l}0.5255 \\
0.3253\end{array}$ & 53.4688 & 110.9376 & 116.1479 & 111.0613 & 4.0611 & 6.1211 \\
& $\hat{\theta}=1.2707$ & 0.0722 & 138.8328 & 279.6656 & 282.2708 & 279.7890 & 1.1292 & 1.4122 \\
\hline AD & $\hat{\theta}=2.3451$ & 0.1541 & 154.8316 & 311.6631 & 314.2683 & 311.7040 & 2.0431 & 2.6471 \\
\hline PAD & $\hat{\alpha}=2.0018$ & 2.0018 & 91.8369 & 187.6739 & 192.8842 & 187.7980 & 1.7984 & 2.2053 \\
& $\hat{\theta}=0.8072$ & 0.0641 & & & & & 0.6816 & 0.9328 \\
\hline
\end{tabular}

Table 4. Estimates of model parameter with standard errors and corresponding values of model selection criteria for the distributions fitted to data set 2

\begin{tabular}{|l|l|l|l|l|l|l|l|l|}
\hline Distribution & $\begin{array}{l}\text { Parameter } \\
\text { Estimates }\end{array}$ & $\begin{array}{l}\text { Standard } \\
\text { Error }\end{array}$ & $\log L$ & AIC & BIC & CAIC & \multicolumn{2}{|c|}{$95 \%$ Conf. Interval } \\
\hline IPAD & $\hat{\theta}=7.1868$ & 1.1768 & 19.9648 & 43.9296 & 48.2160 & 44.1297 & 4.8803 & 9.4933 \\
& $\hat{\alpha}=5.5477$ & 0.5094 & & & & & 4.5493 & 6.5461 \\
\hline AD & $\hat{\theta}=1.2931$ & 0.0928 & 85.6863 & 173.3726 & 175.5157 & 173.4381 & 1.1112 & 1.4750 \\
\hline IAD & $\hat{\theta}=2.3417$ & 0.1938 & 96.2251 & 194.4501 & 196.5932 & 194.516 & 1.9619 & 2.7216 \\
\hline PAD & $\hat{\alpha}=2.3013$ & 0.1494 & 46.5065 & 97.0129 & 101.2992 & 97.2129 & 2.0085 & 2.5941 \\
& $\hat{\theta}=0.7457$ & 0.0779 & & & & & 0.5930 & 0.8984 \\
\hline
\end{tabular}

${ }^{*} L$ and $U$ denote the lower limits of the confidence interval respectively.

Based on the results displayed in Tables 3 and 4 respectively, it is evident that the IPA distribution has the smallest AIC, BIC, CAIC and log-likelihood values among all competing models, and so it could be chosen as the best model among all the distributions which have been fitted to the two data sets. 


\section{Conclusion}

This paper introduced a new two-parameter heavy-tailed distribution called the inverse power Akash distribution and derived some of its properties like moments, mean, variance, mode, coefficient of variation, skewness, kurtosis, moment generating function, quantile function, Rényi entropy, stochastic ordering and the distributions of order statistics. In addition, some functions commonly used in reliability analysis, such as survival, hazard, reversed, cumulative hazard and odds functions respectively have been derived. The model parameters were estimated by using the maximum likelihood estimation procedure. Finally, the proposed model was fitted to two real-life data sets and was compared with the estimates from other extensions of the Akash distribution. The proposed distribution was found provide a better fit than some other competition distributions considered in this study. It is hoped that the proposed distribution will serve as an alternative model to other models available for modelling heavy-tailed data in many areas such as finance, insurance and economics.

\section{Acknowledgements}

The authors would like to thank the reviewers and the editor for their valuable comments and suggestions which improved the quality of the article substantially.

\section{Authors' contributions}

This work was carried out in collaboration among all authors. Samuel U. Enogwe proposed the Inverse Power Akash Distribution and derived its properties, managed the literature searches, wrote the first draft. Happiness O. Obiora-Ilouno carried out the proofreading and ensured proper organization of the paper. Chrisogonus K. Onyekwere performed the analysis. All authors read and approved the final manuscript.

\section{Funding}

The authors' did not receive any specific funding for this work.

\section{Availability of data and materials}

Source of data used in this work have been properly cited and provided in the work.

\section{Competing interest}

The authors have no competing interest. 


\section{References}

[1] R. Shanker, Akash distribution and its applications, Internet. J. Prob. Stat. 4(3) (2015), 65-75. https://doi.org/10.5923/j.ijps.20150403.01

[2] R. Shanker, F. Hagos and S. Sujatha, On modeling of lifetimes data using one parameter Akash, Lindley and exponential distributions, Biom. Biostat. Int. J. 3(2) (2016) 1-10. https://doi.org/10.15406/bbij.2016.03.00061

[3] R. Shanker and H. Fesshaye, On modeling of lifetime data using one parameter Akash, Shanker, Lindley and exponential distributions, Biom. Biostat. Int. J. 3(6) (2016), 1-12. https://doi.org/10.15406/bbij.2016.03.00084

[4] A. Fatima and A. Roohi, Transmuted exponentiated Pareto distribution, Pakistan J. Stat. 32 (2015), 63-80.

[5] R. Shanker, A quasi Akash distribution, Assam Stat. Rev. 30 (1) (2016), 135-160.

[6] R. Shanker and K.K. Shukla, Power Akash distribution and its application, J. Appl. Quant. Meth. 12 (2017), 1-10.

[7] Rama Shanker, K.K. Shukla, Ravi Shanker and A. Pratap, A generalized Akash distribution, Biom. Biostat. Int. J. 7(1) (2018), 18-26.

https://doi.org/10.15406/bbij.2018.07.00187

[8] R. Shanker and K.K. Shukla, On two-parameter Akash distribution, Biom Biostat. Int. J. 6 (2017), 416-425. https://doi.org/10.15406/bbij.2017.06.00178

[9] R.V. Hogg, J.W. McKean and A.T. Craig, Introduction to Mathematical Statistics, 8 ed., Pearson Education, Inc., 2019.

[10] I.S. Olarenwaju and A.A Kazeem, On the Beta-Nakagami distribution, Progress in Appl. Math. 5 (2013), 49-58.

[11] R Core Team, R: A language and environment for statistical computing, R Foundation for Statistical Computing, Vienna, Austria, 2019.

[12] A. Rényi, On measure of entropy and information, in: Proceedings of the 4th Berkeley Symposium on Mathematical Statistics and Probability 1, University of California Press, Berkeley 4 (1961), 547-561.

[13] I.S. Gradshteyn and I.M. Ryzhik, Tables of Integrals, Series, and Products, 7th ed., Elsevier Academic Press, New York, 2007.

[14] D. Cox and D. Hinkley, Theoretical Statistics, Chapman and Hall, London, 1974. https://doi.org/10.1007/978-1-4899-2887-0 
[15] M.R. Mahmoud and R.M. Mandouh, On the transmuted Frechet distribution, J. Appl. Sci. Res. 9 (2013), 5553-5561.

This is an open access article distributed under the terms of the Creative Commons Attribution License (http://creativecommons.org/licenses/by/4.0/), which permits unrestricted, use, distribution and reproduction in any medium, or format for any purpose, even commercially provided the work is properly cited. 